\title{
Alternating asystole and atrial fibrillation after infusion of propofol and remifentanil with target-controlled infusion
}

\author{
Won-jung Hwang, Chan Oh Park, and Yoonki Lee \\ Department of Anesthesiology and Pain Medicine, Seoul St. Mary's Hospital, The Catholic University of Korea, Seoul, Korea
}

Propofol and remifentanil are widely used anesthetics due to rapid onset and offset even after prolonged infusion. However, these agents are known to have vagomimetic effects and may cause conduction delays [1,2]. We describe a case of alternating asystole and atrial fibrillation occurred immediately after start of target-controlled infusion (TCI) of propofol and remifentanil, and late diagnosis of sick sinus syndrome.

A 70-year-old woman (height $165 \mathrm{~cm}$, weight $60.4 \mathrm{~kg}$ ) was scheduled for ventriculo-peritoneal shunt due to hydrocephalus. She had taken aspirin, vasartan and propafenone for hypertension and atrial fibrillation. She had undergone craniectomy due to intracranial hemorrhage about a month previously. During that surgery under general anesthesia with sevoflurane and remifentanil, electrocardiography suddenly changed to supraventricular tachycardia from a normal sinus rhythm about 3 hours after anesthesia induction, and then asystole was followed for a few seconds after intravenous administration of esmolol $10 \mathrm{mg}$. Atropine $0.5 \mathrm{mg}$ was administered intravenously, and electrocardiography restored to a normal sinus rhythm until the end of operation. Postoperative $24 \mathrm{hr}$-Holter electrocardiography showed a normal sinus rhythm with ten paroxysmal atrial fibrillations with three compensatory pauses of less than $3 \mathrm{~s}$. She presented no symptoms associated with arrhythmia, and there were no further treatments.

When routine monitors were applied including the bispectral index, the vital sign was $125 / 85 \mathrm{mmHg}-78 / \mathrm{min}-20 /$ min- $36.7^{\circ} \mathrm{C}$ and $\mathrm{SpO}_{2}$ was $95 \%$. Electrocardiography showed a normal sinus rhythm of $78 / \mathrm{min}$ followed by a sudden change to an atrial fibrillation of $150-160 / \mathrm{min}$, and blood pressure was
170/100 mmHg. To reduce her anxiety, midazolam $2 \mathrm{mg}$ was administered intravenously. Normal sinus rhythm of $80 / \mathrm{min}$ was restored, and blood pressure was 120/80 $\mathrm{mmHg}$. Anesthesia was induced with etomidate $6 \mathrm{mg}$ and rocuronium $40 \mathrm{mg}$, and no change was observed on electrocardiography. The main fluid was infused in the antecubital area at a constant speed of 120 $\mathrm{ml} / \mathrm{hr}$. Propofol and remifentanil were infused for anesthetic maintenance right after the cannulation site of main fluid by a TCI (Orchestra ${ }^{\circledR}$ Base Primea, Fresenius Vial, Brezins, France). The target of infusion was the effect-site concentration; Propofol and remifentanil were infused at $2.0 \mu \mathrm{g} / \mathrm{ml}$ in the Schnider model and $1.5 \mathrm{ng} / \mathrm{ml}$ in the Minto model, respectively. The maximal infusion rate of both drugs was $1,200 \mathrm{ml} / \mathrm{hr}$. The maximal plasma concentrations of propofol and remifentanil were 30.0 $\mu \mathrm{g} / \mathrm{ml}$ and $50.0 \mathrm{ng} / \mathrm{ml}$, respectively. About $1 \mathrm{~min}$ after infusion, electrocardiography revealed atrial fibrillation followed by an asystole of a few seconds. Atropine $0.5 \mathrm{mg}$ and epinephrine 0.1 mg were administered intravenously twice, but no change was noted on electrocardiography, and an alternating arrhythmia of asystole and atrial fibrillation occurred continuously (Fig. 1). A radial artery was cannulated and blood pressure was monitored continuously. Systolic blood pressure was $120-140 \mathrm{mmHg}$ during atrial fibrillation, and unmeasurable during asystole. Arterial blood gas revealed no remarkable electrolyte disturbance. The infusion of propofol and remifentanil was stopped, and electrocardiography restored to a normal sinus rhythm with no additional arrhythmias after approximately $5 \mathrm{~min}$. The infused dose and duration of propofol and remifentanil were $31.6 \mathrm{mg}$ for $20 \mathrm{~s}$ and $22.6 \mu \mathrm{g}$ for $24 \mathrm{~s}$, respectively.

Corresponding author: Yoonki Lee, M.D., Ph.D., Department of Anesthesiology and Pain Medicine, The Catholic University of Korea, Seoul St. Mary's Hospital, 222, Banpo-Daero, Seocho-gu, Seoul 137-701, Korea. Tel: 82-2-2258-6152, Fax: 82-2-537-1951, E-mail: amoeba79@catholic.ac.kr (c) This is an open-access article distributed under the terms of the Creative Commons Attribution Non-Commercial License (http:// creativecommons.org/licenses/by-nc/3.0/), which permits unrestricted non-commercial use, distribution, and reproduction in any medium, provided the original work is properly cited. 


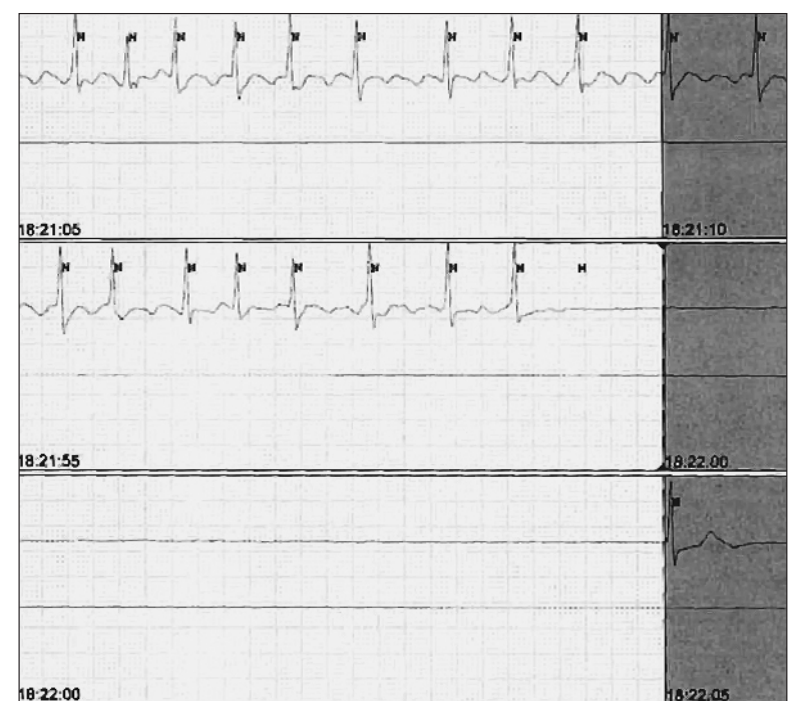

Fig. 1. Alternating arrhythmia of asystole and atrial fibrillation after the admisnitration of propofol and remifentanil by target-controlled infusion.

About 40 min after the cessation of infusion, full recovery of consciousness and self respiration were indentified. Extubation was performed without reversal agents for muscle relaxant after $95 \%$ recovery was confirmed on train-of-four monitor. Postoperative 24 hr-Holter electrocardiography showed 128 paroxysmal atrial fibrillation with 94 compensatory pauses, the longest pause being $6.34 \mathrm{~s}$. She was diagnosed with tachy-bradycardia syndrome, a type of sick sinus syndrome and planned to implant a permanent pacemaker (DDDR mode). After insertion of pacemaker, follow-up $24 \mathrm{hr}$-Holter electrocardiography showed a well-controlled heart rate (average $71 / \mathrm{min}$ ) with rare atrial premature contraction and atrial fibrillation.

Bradycardia associated with propofol or remifentanil in previous reports was mainly associated with old age, existing bradycardia, preoperative medication, a large dose, and a fast speed of injection [1,2]. Our patient was 70 years of age, had taken propafenone due to atrial fibrillation, and compensatory pauses occurred during preoperative $24 \mathrm{hr}$-Holter, although only a small number. Considering these factors, we started the TCI at the lower effect-site concentrations than usual initially and planned to increase the dose of anesthetics incrementally according to the value of the bispectral index. However, we ignored the fact that a rapid bolus was administered at the beginning of infusion by TCI to achieve the effect-site concentration targeted. The initially infused dose of propofol and remifentanil was relatively small considering her weight and age, but the two agents were infused concurrently for a short time. Thus, the concomitant fast administration of propofol and remifentanil could exacerbate existing sinus nodal dysfunction.

TCI is a computerized infusion device for intravenous anesthetic drugs. Based on calculations by pharmacokinetic model, the infusion rate is adjusted to maintain a steady target concentration in the plasma or at the site of drug effect. An initial bolus is necessary to reach the target concentration, and high initial plasma peak concentration is followed. Previous studies found that higher infusion rates of propofol or remifentanil were associated with a significantly higher number of adverse effects $[3,4]$. To reduce adverse effects by the overshoot, limiting the drug infusion by adjustment of maximal flow rate or maximal plasma concentration is suggested [4,5]. In the case of remifentanil loading, the recommended bolus of remifentanil is $1.0 \mu \mathrm{g} / \mathrm{kg} / \mathrm{min}$ over 30-60 s. In present case, the initial infusion rate of remifentanil for initial loading was $2.27 \mu \mathrm{g} / \mathrm{kg} / \mathrm{min}$ for $6 \mathrm{~s}$. If we reduced the maximal flow rate to $100 \mathrm{ml} / \mathrm{hr}$, it might have been $1.11 \mu \mathrm{g} /$ $\mathrm{kg} / \mathrm{min}$ for $20 \mathrm{~s}$ with the same target of effect-site concentration. Even though a prolongation of the time to target might occur, we could reduce the risk of adverse events.

In conclusion, special attention is needed in the administration of anesthetics with vagomimetic effects in patients with advanced age, with preexisting cardiac disease, and who take drugs associated with cardiac conduction. In addition, it may be advocated to optimize or adjust TCI-device setting to avoid administration of a relatively large dose of remifentanil in a short period of time, especially during the initial stage of remifentanil TCI.

\section{References}

1. Thomson SJ, Yate PM. Bradycardia after propofol infusion. Anaesthesia 1987; 42: 430.

2. Reid JE, Mirakhur RK. Bradycardia after administration of remifentanil. Br J Anaesth 2000; 84: 422-3.

3. Bilotta F, Fiorani L, La Rosa I, Spinelli F, Rosa G. Cardiovascular effects of intravenous propofol administered at two infusion rates : a transthoracic echocardiographic study. Anaesthesia 2001; 56: 266-71.

4. Min SK, Kim DH, Cho HB, Moon BK, Kim JY. Limited maximal flow rate of target-controlled remifentanil infusion and induced cough. Anaesthesia 2012; 67: 145-8.

5. Van Poucke GE, Bravo LJ, Shafer SL. Target controlled infusions: targeting the effect site while limiting peak plasma concentration. IEEE Trans Biomed Eng 2004; 51: 1869-75. 